\title{
Los puertos seguros para los intermediarios de internet ${ }^{*}$
}

\author{
Andrés Izquierdo**
}

Recepción: 30 de marzo de 2012 · Aprobación: 5 de junio de 2012

La observancia del derecho de autor es una prioridad en la agenda internacional, pues existe una búsqueda que pretende sustituir las actuales limitaciones de las leyes vigentes a fin de responder a los desafíos de las nuevas tecnologías de la información y la comunicación (TIC). El presente artículo realiza un acercamiento comparado a la legislación concebida para la protección y observancia del derecho de autor en internet de países como Estados Unidos y de la Unión Europea, en relación con la responsabilidad de los intermediarios de internet frente a

* El presente artículo es el resultado de las investigaciones que el autor ha socializado previamente en el marco de la cátedra fray Bartolomé de Las Casas, en la Facultad de Derecho de la Universidad Santo Tomás (abril de 2012).

** Abogado egresado de la Universidad de los Andes, Colombia. Ha cursado seminarios especializados de gestión colectiva de derecho de autor y conexos en la Sociedad General de Autores y Editores de España (SGAE), al igual que seminarios de propiedad intelectual en la Organización Mundial de la Propiedad Intelectual (OMPI), en Ginebra (Suiza). Cursó la Maestría en Propiedad Intelectual en la Universidad de Turín, cuya tesis titulada "Principios de open source aplicado a la biotecnología en Europa" fue laureada, premiada por Microsoft International y publicada por la OMPI en la edición 2006 de Collection of Research Papers WIPO (Turín). Ha ocupado el cargo de asesor jurídico de la Asociación Colombiana de Productores Fonográficos (ASINCOL) y asesor de la Asociación para la Protección de Fonogramas y Videogramas Musicales (APDIF). Es abogado practicante en la firma Frignani \& Associati de Turín. Es socio y director del área de Litigios y Arbitramentos en la firma Palacio \& Ballesteros. Es gerente de Asuntos Legales en Sony Music Entertainment para la región andina. Actualmente es socio y director en el área de Propiedad Intelectual de Vanegas, Izquierdo \& Delgado Abogados. Es investigador en el Insituto Pensar, Labcom, de la Pontifica Universidad Javeriana. Página web: www. andresizquierdo.com - Correo electrónico: info@andresizquierdo.com - Twitter: @andizquierdo 
los contenidos infractores que puedan transitar o alojar en sus sistemas. Se hará un especial énfasis en la legislación actual relativa a los puertos seguros -safe harbours-, en la jurisprudencia que ha tratado de modelar este comportamiento y en los nuevos estatutos que se han generado como respuesta a una búsqueda de mayor protección y observancia del derecho de autor en internet.

Palabras clave: Observancia del derecho de autor, tecnologías de la información, intermediarios de internet, puertos seguros, notificación, retiro de contenidos. 


\section{Safe Harbors for Online Service Providers}

Abstract

Copyright enforcement is an international agenda priority; there is a search to replace the current limitations of existing laws to respond to the challenges of new digital technologies. This article makes a comparative approach to the legislation designed to protect and enforce copyright on the Internet in countries such as the United States of America and the European Union, regarding the responsibility of Internet intermediaries for the infringing content that may travel through or stay in their systems. The article will make special emphasis on current legislation relating to the safe harbors, on jurisprudence that has tried to model this digital behavior, and on new legislative models that seek a higher protection and copyright enforcement on the internet.

Keywords: Copyright enforcement, digital technologies, Internet, safe harbors, notice, content withdrawal. 


\section{Les sphères de sécurité pour les intermédiaires d'Internet}

Le respect du droit d'auteur constitue une priorité dans l'agenda internationale, il existe même une recherche pour substituer les limitations actuelles des lois en vigueur afin de répondre aux défis des TIC. Cet article fait un rapprochement comparatif de la législation conçue pour la protection et le respect du droit d'auteur sur Internet auprès de pays tels les États-Unis et les pays de l'Union Européenne par rapport à la responsabilité des intermédiaires de l'Internet face aux contenus infracteurs pouvant passer ou loger dans leurs systèmes. Nous insisterons notamment sur la législation actuelle concernant les sphères de sécurité, sur la jurisprudence qui a essayé de façonner ce comportement, et sur les nouveaux statuts générés en tant que réponse à une recherche de plus de protection et de respect du droit d'auteur sur Internet.

Mots-clés: Respect du droit d'auteur, technologies de l'information, intermédiaires de l'Internet, sphères de sécurité, notification, élimination de contenus. 


\section{Introducción}

Es claro que la observancia de los derechos de propiedad intelectual es una prioridad en la agenda internacional, pues existe una búsqueda que pretende sustituir las actuales limitaciones de las leyes vigentes a fin de responder a los desafíos de las nuevas tecnologías de la información y la comunicación. En la historia del derecho de autor, cuando de tecnología se trata, siempre ha existido controversia respecto a su contenido, alcance, métodos de ejecución y protección. Desde la creación de la imprenta, pasando por los rollos de piano, la radio, la televisión por cable, el betamax, hasta las últimas aplicaciones P2P, todos ellos hacen que el enfrentamiento entre las nuevas tecnologías y el derecho de autor se repita en numerosas ocasiones.

Ante los nuevos desafíos del derecho de autor, han surgido modelos legislativos que tratan de responder preguntas de este tipo: ¿cómo hacer cumplir los derechos de autor en internet?, ¿qué aplicación tiene el derecho internacional privado? Lo anterior ha llevado a otros interrogantes: ¿en qué medida la legislación autoral compromete los derechos humanos?, ¿cómo se soluciona el conflicto entre el derecho de autor cuando se ve enfrentado a la libertad de expresión, la protección de datos y las leyes de privacidad? Y en cosas más técnicas surgen cuestionamientos como los siguientes: ¿cómo se recaudan las pruebas?, ¿quién está en infracción: el que sube, el que descarga o el que presta sus servicios digitales de comunicaciones?

El presente artículo busca hacer un acercamiento a la legislación concebida para la protección y observancia del derecho de autor en internet de países como Estados Unidos y de la Unión Europea, en relación con la responsabilidad de los intermediarios de internet ${ }^{1}$ frente a los contenidos infractores que puedan transitar o alojar en sus sistemas. Se hará

1 La Organización para el Desarrollo y Cooperación Económica (OECD) define a los intermediarios de internet como aquellos que otorgan acceso a hosting, que transmiten o almacenan contenidos originados por terceros o que proporcionan servicios de internet a estos. Ejemplos de intermediarios son los proveedores de servicios de internet (ISP), de almacenamiento, de hosting, de motores de busqueda y paginas web, de sistemas de pago, de e-commerce, de plataformas de redes sociales, entre otros. Las legislaciones se refieren, en numerosas ocasiones, a los intermediarios de internet como los prestadores de servicios o service provider. Al respecto, la Directiva 2000/31/CE define "prestador de servicios" como cualquier persona física o jurídica que suministre un servicio de la sociedad de la 
un especial énfasis en la legislación actual relativa a los puertos seguros -safe harbours-, en la jurisprudencia que ha tratado de modelar este comportamiento y en los nuevos estatutos que se han generado como respuesta a una busqueda de mayor protección y observancia del derecho de autor en internet.

\section{Contexto histórico de la observancia del derecho de autor y su transición a la protección en internet}

La evolución de las normas internacionales para la observancia del derecho de autor ha sido notable en los últimos años; cambio que ha sido impulsado principalmente por dos factores: a) el avance de los nuevos medios tecnológicos para la creación y uso de obras protegidas en internet ${ }^{2}$, incluyendo el advenimiento de nuevos desarrollos digitales que permiten transmitir y hacer copias perfectas de cualquier información existente, entre las que se encuentran aquellas obras y producciones protegidas por derechos de autor y derechos conexos; b) la creciente importancia económica de los bienes y servicios ofrecidos en internet en el ámbito del comercio internacional, en el cual, sin duda alguna, tienen un papel muy representativo los derechos de propiedad intelectual ${ }^{3}$.

Al revisar la normatividad vigente es claro que existen numerosos tratados internacionales, acuerdos multilaterales y nuevas legislaciones nacionales que buscan formas de proteger el derecho de autor ${ }^{4}$. Entre las primeras

información. Por su parte, en Estados Unidos se refieren a los intermediarios como service providers, y en China como network service providers. Para mayor información visítese: http://www.oecd.org/

2 Conforme a este documento, los usuarios de internet en todo el mundo llegaron a 1,7 billones a finales de septiembre de 2009, lo que significa que más de un cuarto de la población mundial tiene acceso a internet. China tiene la mayor audiencia de internet en el mundo, con 360 millones de usuarios, seguido por Estados Unidos (230 millones), Japón (100 millones), Alemania (54 millones) y Reino Unido (47 millones) (OECD, 2010, p. 9).

3 En un censo realizado en Estados Unidos en el 2008, respecto a los ingresos de los intermediarios en internet (ISP, buscadores, e-commerce, páginas web, entre otros que se nombraran más adelante en el presente artículo), se encontró que: a) los ISP obtuvieron ingresos por 68 billones de dólares en el 2008 (un $12 \%$ más que el año anterior); b) para el procesamiento de datos, hosting y servicios relacionados se obtuvo un ingreso de 78 billones de dólares (un 2,9\% más que el año anterior); c) los portales web mostraron ingresos por 14 billones de dólares en 2008 (es importante resaltar que obtuvieron un 19\% más que el año anterior). Al respecto véase: www.oecd.org/dataoecd/49/4/44949023.pdf

4 En el presente texto entiéndase "derecho de autor" como "copyright" para las normas de tradición anglosajona; y "derecho de autor y conexos" para las normas de tradición continental. 
normas concebidas para tal efecto se puede encontrar el Convenio de Berna ${ }^{5}$, el cual contiene dos disposiciones específicas sobre la observancia de derecho de autor en su artículo 16, donde se establece: "1) Toda obra falsificada podrá ser objeto de decomiso en los países de la Unión en que la obra original tenga derecho a la protección legal; 2) Las disposiciones del párrafo precedente serán también aplicables a las reproducciones procedentes de un país en que la obra no esté protegida o haya dejado de estarlo". Por su parte, el artículo 13.3 del mismo Convenio prevé la confiscación de las copias de las grabaciones de algunas de las obras musicales importadas sin autorización de las partes interesadas, en un país en que estas grabaciones no sean lícitas (Organización Mundial de la Propiedad Intelectual [OMPI], 2004, p. 231).

Los Convenios de Berna, Roma ${ }^{6}$ y de Fonogramas ${ }^{7}$ también contienen disposiciones que indirectamente requieren medidas adecuadas para la protección de estos derechos en cualquier país parte en las convenciones. El artículo 36.1 del Convenio de Berna establece: "Todo país que forme parte del presente Convenio se compromete a adoptar, de conformidad con su Constitución, las medidas necesarias para asegurar la aplicación del presente Convenio". El párrafo segundo del mismo artículo señala: "Se entiende que en el momento en que un país se obliga por este Convenio, se encuentra en condiciones, conforme a su legislación interna, de aplicar las disposiciones del mismo". Disposiciones similares se encuentran en el artículo 26.1 y 26.2 del Convenio de Roma, en el que se exige a los Estados contratantes a tomar las medidas necesarias para garantizar la aplicación de la Convención. Por su parte, el artículo 2 del Convenio de Fonogramas obliga a cada Estado contratante a proteger a los productores de fonogramas contra la producción de duplicados (copias) sin el consentimiento de los productores y contra la importación y distribución de esas copias. Finalmente, el artículo 3 de la Convención deja la implementación del

5 El Convenio de Berna para la Protección de las Obras Literarias y Artísticas fue expedido inicialmente el 9 de septiembre de 1886, revisado y completado varias veces y enmendado por última vez el 28 de septiembre de 1979.

6 Convención Internacional sobre la Protección de los Artistas Intérpretes o Ejecutantes, los Productores de Fonogramas y los Organismos de Radiodifusión; hecho en Roma el 26 de octubre de 1961.

7 Convenio para la Protección de los Productores de Fonogramas contra la Reproducción no Autorizada de sus Fonogramas; hecho el 29 de octubre de 1971. 
convenio a las partes contratantes, en los casos en que la debida protección se puede ejercer por medio del derecho de autor u otros específicos, lo que implica considerar la competencia desleal y las sanciones penales correspondientes.

Sin embargo, la efectividad de los anteriores tratados, en términos de observancia, se vio cuestionada en los años ochenta, situación que generó el estudio y preparación de nuevas y obligantes normas internacionales. Así las cosas, en 1986, durante la Ronda de Uruguay, se introdujeron en el General Agreement on Tariffs and Trade (GATT) negociaciones multilaterales para los derechos de propiedad intelectual relacionados con el comercio. En la discusión, los titulares de los derechos de propiedad intelectual, así como los países industrializados, hicieron presión a fin de obtener un régimen legal internacional para la efectiva prohibición del comercio que infringiera estos derechos (Correa, 2001, p. 1). Estas demandas culminaron y formaron, en última instancia, la base para el Acuerdo sobre los Aspectos de los Derechos de Propiedad Intelectual relacionados con el Comercio (ADPIC), el cual fue adoptado en el anexo $1 \mathrm{C}$ del Convenio por el cual se creó la Organización de Comercio Mundial (OMC), firmado en Marrakesh en el año de 1994.

Luego de la adopción de los $\mathrm{ADPIC}^{8}$ se intensificó el trabajo preparatorio en los comités de la OMPI para la creación de nuevas normas en derecho de autor y derechos conexos, a fin de tratar problemas no incluidos en mencionado acuerdo (OMPI, 2004, p. 269). Así, para el año de 1996 se promulgaron el Tratado de la OMPI sobre Derecho de Autor (WCT) y el Tratado de la OMPI sobre Interpretación o Ejecución y Fonogramas (WPPT), conocidos ambos como los Tratados Internet de la OMPI. Estos tratados son considerados como actualizaciones y complemento de la protección garantizada por el Convenio de Berna y la Convención de Roma. Buscan solucionar los inconvenientes de la observancia del derecho de autor y derechos conexos, generados a partir de los avances en las

$8 \quad$ Al respecto véase: Izquierdo y Palacio (2011); artículo en el que se hace un estudio comparativo de la implementación legal de los ADPIC en las legislaciones de la Unión Europea, de los Estados Unidos de América y de Colombia. 
tecnologías digitales y, en particular, en la diseminación de contenidos protegidos en internet ${ }^{9}$.

Las disposiciones contenidas en ambos tratados -cada una dentro de su especialidad-incluyen normas aplicadas al almacenamiento y transmisión de obras, interpretaciones y fonogramas en sistemas digitales, al igual que señalan las limitaciones y excepciones a los derechos en el entorno digital y las medidas tecnológicas de protección y gestión de derechos. Estos tratados son de suma importancia para la legislación actual mundial, toda vez que los países signatarios ${ }^{10}$ ratificaron, promulgaron y/o implementaron nuevas normas de protección para los contenidos en los entornos digitales. De allí nacen estatutos como la Digital Millenium Copyright Act (DMCA) ${ }^{11}$ de Estados Unidos, o la Directiva 2000/29/EC ${ }^{12}$ de la Comunidad Económica Europea. Estos últimos modelos legislativos han sido adoptados, cada uno a su medida, por casi cuarenta países, incluyendo los veintisiete miembros de la Unión Europea, Australia, China, Japón y Singapur. Países como Corea del Sur, España, Francia, Malasia, Nueva Zelanda y el Reino Unido han implementado estatutos complementarios y más estrictos en el cumplimiento de estas normas.

Frente a lo anterior, uno de los temas preponderantes y que ha cobrado mayor importancia en los últimos años en diferentes legislaciones es la actuación de los intermediarios de internet para la observancia del derecho de autor. En el mismo sentido, no han sido escasas las decisiones judiciales que han exigido e imputado responsabilidad a los proveedores de servicio de internet (ISP), a los proveedores de espacio de almacenamiento o a los editores de programas peer-to-peer, a causa de la no observancia

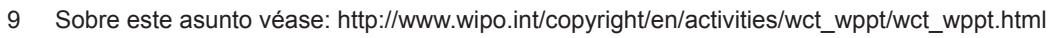

10 La lista de las partes firmantes del WCT puede verse en http://www.wipo.int/treaties/es/ShowResults. jsp?lang=es\&treaty_id=16. Por su parte, las partes signatorias del WPPT puede consultarse en http:// www.wipo.int/treaties/en/notifications/wppt/treaty_wppt_1.html. Colombia hace parte de estos tratados ratificados por medio de la Ley 545 de 1999 y Ley 565 de 2000.

11 La parte inicial de la DMCA, con registro H.R.2281 de 1998, establece: "An Act, to amend title 17, United States Code, to implement the World Intellectual Property Organization Copyright Treaty and Performances and Phonograms Treaty, and for other purposes. Be it enacted by the Senate and House of Representatives of the United States of America in Congress assembled". El texto puede verse en línea: http://www.gpo.gov/fdsys/pkg/BILLS-105hr2281enr/pdf/BILLS-105hr2281enr.pdf

12 Algunas de las Directivas que complementan los Tratados son: Directiva 91/250/EC sobre software, Directiva 96/9/EC sobre Bases de Datos y Directiva 2000/31/EC sobre Comercio Electrónico. 
adecuada de estos derechos. Casos judiciales como los de RapidShare, Tiscali, Myspace o Dailymotion son solo algunos ejemplos recientes que identifican cuáles podrían ser, por mandato legal o sentencia judicial, los nuevos protectores del derecho de autor en los medios digitales ${ }^{13}$.

\begin{abstract}
A continuación se revisarán los desarrollos legales respecto a la responsabilidad de los intermediarios de internet frente a la observancia de los derechos de autor en internet, en los casos de Estados Unidos de América ${ }^{14}$ y los países de la Unión Europea ${ }^{15}$. Se hará un especial énfasis en la legislación actual relativa a los puertos seguros -safe harbours- en internet y en la jurisprudencia que ha tratado de modelar este comportamiento.
\end{abstract}

\title{
Responsabilidad de los intermediarios de internet
}

\begin{abstract}
Hay un tema recurrente en las legislaciones de Estados Unidos y de la Unión Europea -salvo algunas nuevas normas que se estudiarán más adelante en este escrito- que obliga a los intermediarios que proporcionan
\end{abstract}

13 Es necesario subrayar que el problema de la observancia del derecho de autor en internet se presenta en el mundo entero y ha dado lugar a diversas respuestas judiciales. Véanse los inicios con los siguientes casos norteamericanos: U.S. Court of Appels for the Ninth Circuit (2001). A\&M Records vs. Napster, 239 F.3d 1004; U.S. Supreme Court (2005). 9th Cir. 201; (Metro-Goldwyn-Mayer Studios vs. Grokster, 125, S. Ct. 2764. También puede verse el caso Kazaa (2005). F. C. A. 1242, en Australia; y, más recientemente, el caso Pirate Bay en Suecia (Svea hovrätt), del 26 de noviembre de 2010 (No. B 4041-09).

14 En el momento de redacción del presente artículo se encontraba en estudio en Estados Unidos la Stop Online Piracy Act (SOPA), la cual, en el proyecto presentado inicialmente en el Congreso de Estados Unidos, buscaba principalmente lo siguiente: a) el proyecto de ley permitiría al Departamento de Justicia de los Estados Unidos conseguir órdenes judiciales contra sitios web acusados de infringir los derechos de autor, o de permitir o facilitar la infracción de los derechos de autoría; b) el fiscal general podría instar a los motores de búsqueda en internet, tales como Google o Yahoo!, a que eliminen los enlaces que vinculen con tales sitios; c) se requeriría una posición activa por parte de los intermediarios de internet; d) luego de entregar la orden judicial, el Fiscal general de los Estados Unidos podría requerir a aquellos proveedores de internet que ofreciesen sus servicios en el territorio de Estados Unidos, a sus redes de publicidad asociadas y a los facilitadores de pago online que suspendieran sus negocios con los sitios que fuesen encontrados en infracción criminal de las leyes federales de propiedad intelectual; d) podría darse cárcel y sanciones para las personas que subieran contenidos no autorizados (por ejemplo Youtube). En términos generales, el proyecto de ley generó una fuerte oposición, liderada principalmente por Google, Yahoo!, Facebook, Twitter, AOL, Linkedln, eBay, Mozilla Corporation, Reddit, la Fundación Wikimedia; al igual que por organizaciones ambientalistas y de derechos humanos, tales como Greenpeace, Reporteros Sin Fronteras, Electronic Frontier Foundation, ACLU y Human Rights Watch. Esta ley modificaría la Digital Millenium Copyright Act de 1998.

15 Para los fines del presente escrito, cuando se hace referencia a las normas de la Unión Europea se debe entender desde las directivas supranacionales dictadas por su órgano legislativo; no se debe interpretar como una referencia a las normas internas de cada país perteneciente a la Comunidad. 
la transmisión o el alojamiento web para actuar pasivamente y de forma neutral hasta tanto no les sea comunicada una presunta vulneración de derecho de autor por parte de su titular. También se encuentra que ninguna de las normas estudiadas obliga a los intermediarios a supervisar el contenido que se transmite o se aloja, como tampoco existe algún estatuto en que se les exija realizar esfuerzos para evitar el contenido infractor de derecho de autor que se aloje o transite a través de sus redes (DeBeer \& Clemmer, 2008, p. 375) ${ }^{16}$.

Lo anterior se puede definir como el régimen de responsabilidades de los intermediarios de internet, en donde las leyes de cada país determinan en qué momento son responsables o no del contenido que transita por sus redes. Es así que se genera la política de los puertos seguros -o safe harbours- en internet ${ }^{17}$, por medio de la cual se exonera de responsabilidad a los intermediarios frente a los contenidos infractores de sus usuarios, a condición de que se preste cabal cumplimiento de las normas definidas. En las leyes estudiadas, esta responsabilidad se determina principalmente de acuerdo con el comportamiento ejercido por el intermediario de internet respecto de las comunicaciones digitales transitorias, el alojamiento web o hosting y el almacenamiento de caché o catching. Este enfoque se puede apreciar en las leyes de Estados Unidos ${ }^{18}$ y de los países de la Unión Europea ${ }^{19}$.

\section{Comunicaciones digitales transitorias}

Las comunicaciones transitorias se definen como aquella información que se transmite en una red de comunicaciones entre los destinatarios de un

16 Veáse: DeBeer \& Clemmer (2009). Global trends in online copyright enforcement: a non neutral role for network intermediaries? Puede verse el resumen del artículo en http://ssrn.com/abstract=1529722. En este documento, los autores analizan la incidencia de las nuevas normas mundiales del derecho de autor en el régimen de las comunicaciones. Al respecto, es relevante examinar escritos de Tim Wu acerca de las leyes de comunicaciones y el derecho de autor en Estados Unidos.

17 Los puertos seguros o safe harbours no son de exclusiva aplicación para las normas de copyright, y se pueden encontrar disposiciones similares en Estados Unidos para el derecho comercial, derecho tributario o derecho financiero.

18 Digital Millennium Copyright Act (2006). 17 U.S.C., § 512. Puede consultarse en http://thomas.loc.gov/ cgi-bin/query/z?c105:H.R.2281

19 Parlamento Europeo y del Consejo (2000, 17 de julio). Directiva 2000/31/CE, artículo 13(e). Diario Oficial No. L178. Recuperado de http://eur-lex.europa.eu/LexUriServ/LexUriServ.do?uri=CELEX: 32000L0031:Es:HTML 
determinado servicio. La mayoría de los sistemas legales en que se contemplan normas relativas a los puertos seguros tratan, de alguna forma, la responsabilidad en las comunicaciones digitales transitorias para los intermediarios de internet (DeBeer y Clemmer, 2009, p. 379). Esta clase de normas exoneran de responsabilidad a los anteriores intermediarios frente a todo tipo de comunicaciones, incluso en los contenidos infractores de derecho de autor que puedan pasar a través de sus redes, en tanto sus comportamientos se sujeten a determinadas condiciones. En general, el intermediario no será responsable de la operación siempre y cuando el material objeto de la infracción se transmita por solicitud de un tercero a un destinatario, la transmisión de esos datos sea manejada por un proceso automatizado sin intervención humana y los datos no se modifiquen en modo alguno, sino que solo se almacenen temporalmente en el sistema.

La Directiva 2000/31 de la Unión Europea, en su artículo 12.1 y 12.2 trata puntualmente el tema y establece que no se puede considerar al intermediario de internet como responsable de los datos transmitidos por el destinatario del servicio, en tanto dicho intermediario cumpla las siguientes condiciones: a) no origine la transmisión; b) no seleccione al destinatario de la transmisión; y c) no seleccione ni modifique los datos transmitidos. Las nombradas actividades de transmisión y concesión de acceso engloban el almacenamiento automático, provisional y transitorio de los datos transmitidos, siempre que dicho almacenamiento sirva exclusivamente para ejecutar la transmisión en la red de comunicaciones y que su duración no supere el tiempo razonablemente necesario para dicha transmisión.

Un similar desarrollo se puede encontrar en la legislación de Estados Unidos ${ }^{20}$, en la cual se establecen varios requisitos de eximentes de responsabilidad para los intermediarios, entre los cuales, de forma no taxativa, se pueden observar los siguientes: a) se exige que una persona diferente al intermediario haya iniciado la transmisión; b) la comunicación debe ser llevada a cabo por un proceso automatizado, sin que haya

20 Ver: United States Code, Tittle 17, Section 512(a); disposición que establece un puerto seguro para los intermediarios que ofrezcan los siguientes servicios: "transmission, routing, or providing of connections for digital online communications, between or among points specified by a user, of material of the user's choosing, without modification to the content of the material as sent or received". 
selección de contenidos por parte del intermediario; c) el intermediario no debe determinar el destinatario de la transmisión; d) las copias solo se pueden conservar durante el tiempo que sea razonablemente necesario; y e) el intermediario no debe modificar el material de ninguna manera ${ }^{21}$.

En Europa, los tribunales de justicia han avocado demandas relacionadas directa o indirectamente con el artículo 12 de la Directiva 2000/31, en casos como el de la Sociedad de Autores y Compositores de Bélgica (SABAM) contra el ISP Tiscali (Scarlet), o el de la Sociedad de Autores de Alemania (GEMA) contra RapidShare, sitio web de intercambio de archivos. Estos casos han dado como resultado una serie de obligaciones en cabeza de los intermediarios de internet condenados judicialmente, lo que los hace responsables de la vigilancia de los contenidos infractores de derecho de autor que transitan por sus redes o que se archivan en sus sistemas.

Al respecto, en junio del 2007, un tribunal belga ${ }^{22}$ ordenó a un proveedor de servicios de internet instalar un software de filtrado, a fin de identificar y bloquear el intercambio ilegal de archivos de música protegidos por el derecho de autor. Esta fue la primera de varias decisiones judiciales similares en Europa, y fue el resultado de una demanda interpuesta por la asociación que representa a los autores y compositores en Bélgica, SABAM, contra el proveedor de servicio de internet Tiscali; acción que se centró en la posibilidad de implementar tecnologías con el propósito de bloquear o filtrar infracciones peer-to-peer (P2P) en las redes del ISP demandado. El Tribunal de Primera Instancia de Bruselas ordenó al ISP Tiscali adoptar, en el plazo de seis meses, una de las once medidas técnicas establecidas en el informe de un experto designado por el tribunal, a fin de prevenir la descarga ilegal de música en sus redes ${ }^{23}$.

21 Al respecto vale señalar que las empresas de telefonía celular en Estados Unidos que ofrecen servicios de internet para sus dispositivos móviles establecen cláusulas en sus contratos de suscripción, como la siguiente: "Como proveedor de comunicaciones digitales transitorias, las actividades de Sprint están protegidas por la disposición de protección legal de la DMCA (ver U.S.C. 512 [a]). Por lo tanto, Sprint no está obligado a responder ante un propietario de derechos de autor (o ante el agente del propietario), ni Sprint tiene la obligación de retirar o inutilizar el acceso a materiales transmitidos, dirigidos o conectados a la(s) red(es) de Sprint que son iniciados y/o dirigidos por un usuario individual". pub2010/2010\%2001\%2028-tiscali-scarlet.pdf 
La sentencia de primera instancia plantea dos inquietudes: a) cambia el requisito de neutralidad establecido en el artículo 15 de la Directiva $2000 / 31^{24}$, al imponer una labor de vigilancia sobre los contenidos que transitan en sus redes; b) el fallo parece estar en conflicto con el artículo 12 de la Directiva, al establecer nuevas condiciones para otorgar inmunidad de puerto seguro a los ISP cuando presten sus servicios como intermediarios. Aunque la decisión actualmente se encuentra en apelación, de confirmarse la sentencia contra el ISP demandado, podría implicar que decisiones similares se impondrían contra los otros ISP de Bélgica, que tendrían la obligación legal de implementar las medidas técnicas contra el intercambio ilegal de archivos.

La decisión apelada por Tiscali fue enviada al tribunal de apelación de Bruselas. Dadas las implicciones del caso, este tribunal a su vez decidió suspender el procedimiento y plantear al tribunal de justicia de Luxemburgo las siguientes cuestiones prejudiciales ${ }^{25}$ : a) responder si las directivas de la Comunidad Europea permiten autorizar a un juez nacional para que ordene a un proveedor de servicios de internet que establezca, con respecto a toda su clientela, un sistema de filtrado de todas las comunicaciones electrónicas que circulen a través de sus servicios, en particular mediante la utilización de programas peer-to-peer, con el fin de identificar en su red la circulación de archivos infractores de derecho de autor y, posteriormente, bloquear la transmisión de dichos archivos; b) en caso de respuesta afirmativa a la primera cuestión planteada al tribunal de Luxemburgo, ¿obligan dichas directivas a un juez nacional, que conoce de una solicitud de mandamiento judicial de cese frente a un intermediario y cuyos servicios son utilizados por un tercero para vulnerar derechos de autor, a aplicar el principio de proporcionalidad al pronunciarse sobre la efectividad y el efecto disuasorio de la medida solicitada?

24 La Directiva 2000/31 en su artículo 15.1 establece la inexistencia de obligación general de supervisión para los proveedores de servicios: "Los Estados miembros no impondrán a los prestadores de servicios una obligación general de supervisar los datos que transmitan o almacenen, ni una obligación general de realizar búsquedas activas de hechos o circunstancias que indiquen actividades ilícitas, respecto de los servicios contemplados en los artículos 12,13 y $14 "$ ".

25 Véanse las recomendaciones del abogado Pedro Cruz Villalón, presentadas al Tribunal de Justicia de Luxemburgo en el mes de abril de 2011: http://eur-lex.europa.eu/LexUriServ/LexUriServ.do?uri=C ELEX:62010CC0070:ES:HTML\#Footref7 
El tribunal de Luxemburgo ${ }^{26}$ todavía no se ha pronunciado, pero dada la estructura de las normas de la Union Europea, es posible que responda en sentido negativo a la primera de las cuestiones prejudiciales planteadas por el tribunal de apelaciones de Bruselas y, en consecuencia, declare que tampoco procede responder a la segunda cuestión. Entre los argumentos de mayor peso para absolver a Scarlet se encuentra que el sistema de filtrado exigido por SABAN está abocado, con independencia de su modo concreto de funcionamiento, a aplicarse con carácter sistemático y universal, continuado y permanente, pero sin que su implantación vaya acompañada de garantía específica alguna en lo que atañe a la protección de los datos personales y a la confidencialidad de las comunicaciones. Estos últimos aspectos están consagrados en la Carta de los Derechos Fundamentales de la Unión Europea, así como en el Convenio Europeo para la Protección de los Derechos Humanos y de las Libertades Fundamentales (CEDH).

En otro caso muy similar, la Sociedad Alemana de Derechos de Autor (GEMA, por su nombre en la lengua propia) presentó una demanda en enero de 2008 contra la empresa RapidShare ante el tribunal de distrito de Düsseldorf, argumentando que el sitio era legalmente responsable de la carga y descarga no autorizadas de obras de los miembros de la sociedad de gestión. RapidShare es una empresa alemana con sede en Suiza y es uno de los sitios web de intercambio de archivos más visitados en todo el mundo. El sitio permite a los usuarios subir archivos, a los cuales se le proporciona una URL única por cada archivo cargado, y luego el usuario puede compartir la URL con quien desee descargar el archivo.

El tribunal de Düsseldorf, aparentemente siguiendo el razonamiento de la Corte de Bélgica en el caso SABAM, ordenó a RapidShare tomar medidas obligatorias para evitar en su sistema la carga y descarga de las

26 A la fecha de redacción del presente artículo no existía un fallo judicial. Al respecto, el presente asunto ofrece al tribunal de justicia la oportunidad de conocer, en un primer momento, sobre la cuestión de las infracciones de derechos de autor y derechos afines cometidas en internet por las descargas ilegales de obras protegidas. Más concretamente, el tribunal de justicia está llamado a pronunciarse por primera vez sobre la viabilidad, desde el punto de vista del derecho de la unión, de determinadas medidas técnicas para la observancia de estos derechos. Veáse el caso Promusicae (Sentencia de 29 de enero de 2008), en que se pedía a un proveedor de acceso a internet, en el marco de un proceso judicial, que revelara la identidad y la dirección de personas identificadas por su dirección IP, así como las fechas y las horas de sus conexiones. 
obras representadas por la sociedad demandante. Dado que RapidShare aloja más de 15 millones de archivos de música, el cumplimiento de la sentencia del tribunal presenta importantes desafíos y, en consecuencia, RapidShare se vería en obligación de cesar sus operaciones. RapidShare también apeló la decisión y todavía no hay confirmación o rechazo de la sentencia de primera instancia. Sin embargo, es claro que el concepto prejudicial del tribunal de justicia de Luxemburgo influirá en la decisión final del tribunal alemán.

\section{Hosting}

El hosting o alojamiento web es el servicio que provee a los usuarios de internet un sistema para poder almacenar información, imágenes, video o cualquier contenido accesible vía web. El hosting ${ }^{27}$ es un tipo de servicio de alojamiento en internet que permite a los individuos y organizaciones hacer accesibe su propio sitio web a través de internet. Las empresas que prestan este servicio -también denominadas web hosts- son compañías que otorgan espacio en un servidor, de su propiedad o en arrendamiento, para uso de sus clientes; en algunos casos también proporcionan conectividad a internet.

Es importante la anterior diferenciación, toda vez que en la actualidad los proveedores de servicios de internet no son los únicos que pueden prestar el servicio de hosting, pues existen nuevos intermediarios que ofrecen servicios -como el alojamiento gratuito- para que sus suscriptores puedan abrir páginas web vinculadas y cargar contenidos. Es así que intermediarios de internet como los blog hosting (ej.: Wordpress, Blogger), los video hosting (ej.: Youtube, Vimeo, Myspace) y los image hosting (ej.: Flicker, Picasa) ofrecen servicios para que sus suscriptores carguen los contenidos relacionados con la finalidad de la página; situación que ha llevado a algunos de estos intermediarios a enfrentar demandas por

27 Actualmente existen diferentes clases de alojamiento web hosting; entre las más utilizadas se encuentran las siguientes: free web hosting service, shared web hosting service, reseller hosting service, virtual dedicated server, dedicated hosting service, managed hosting service, blog hosting service, video hosting service, image hosting service y cloud hosting service. Youtube, al ser un video hosting service, ha tenido numerosas reclamaciones legales por videos infractores de derecho de autor cargados por sus usuarios. 
violación de derechos de autor ante tribunales judiciales en diferentes partes del mundo.

En las legislaciones examinadas, en términos generales se puede observar que la responsabilidad legal de los intermediarios se sujeta normalmente a los siguientes tres contextos: a) tener o no conocimiento de las actividades ilícitas; b) haber recibido notificación de presuntas infracciones y tomar o no las medidas correspondientes para el retiro de los contenidos; y c) tener o no beneficios económicos de la infracción. En la mayoría de las legislaciones hay al menos dos condiciones para eximir de responsabilidad a los intermediarios de internet por hospedar en sus sistemas algún tipo de material infractor de derecho de autor (DeBeer \& Clemmer, 2008, p. 384).

En primer lugar, los intermediarios de internet no deben tener ningún conocimiento real de la presencia de contenido infractor de derecho de autor en sus sistemas. En segundo lugar, si los intermediarios son alertados por denuncias de violación de derecho de autor, deben actuar rápidamente para remover o deshabilitar el acceso a este, lo que se conoce como el notice and takedown o "notificación y retiro de contenido presuntamente infractor". Bajo tal sistema, a los titulares de los derechos de autor se les permite ponerse en contacto con el intermediario de internet para hacer la correspondiente denuncia por infracción de material autoral. Una vez que este aviso ha sido recibido, el intermediario tiene la obligación de eliminar el contenido que es objeto de la reclamación; el no hacerlo resultará en una pérdida de las protecciones otorgadas en el puerto seguro.

La Directiva 2000/31 de la Unión Europea, en el artículo 14.1 (literal a y b), es clara en establecer todos los requisitos de exoneración de responsabilidad de los intermediarios de internet, requiriendo que este no tenga conocimiento efectivo de actividades o contenidos ilícitos, al igual que en lo referente a una demanda por daños y perjuicios no tenga conocimiento de hechos o circunstancias por los que la actividad o la información pueda ser ilícita. Adicionalmente se le exige que en cuanto tenga conocimiento de estos puntos, el intermediario de internet actúe con prontitud para retirar los datos o hacer que el acceso a ellos sea imposible. 
Por su parte, en la legislación de los Estados Unidos ${ }^{28}$ se establece que el intermediario de internet se exonera de responsabilidad por el contenido infractor de derecho de autor almacenado en la dirección de un usuario que se encuentre en su sistema o red, siempre y cuando: a) no tenga conocimiento efectivo de que el contenido infractor o una actividad que usa el contenido infractor opere en su sistema o su red; b) a falta de conocimiento real de la infracción, desconozca los hechos o circunstancias por los que la actividad infractora es aparente; c) en el momento de tener conocimiento de la presunta infracción actúe con prontitud para retirar o bloquear el acceso del contenido infractor; y d) no reciba un beneficio económico directamente atribuible a la actividad infractora.

Al respecto se debe mencionar que la protección del derecho de autor en relación con el hosting no ha sido pacífica a nivel jurisprudencial, pues numerosos casos han sido adelantados ante los tribunales judiciales en el mundo. En los últimos años se han presentado varias reclamaciones y demandas contra proveedores de servicios de hosting como Youtube, Youkioske, MySpace y DailyMotion; casos que han sido adelantados principalmente en tribunales europeos y de Estados Unidos.

Así, en junio de 2007 un humorista francés demandó con éxito a MySpace ${ }^{29}$, por infringir su derecho de autor y derecho de la personalidad, después de que varios de sus sketches fueron publicados por un usuario en una de las páginas que aloja el sitio web. El tribunal francés de primera instancia consideró a MySpace como un editor, a pesar del hecho de que el sitio lleva a cabo las tareas técnicas de un servicio de alojamiento web. La Corte señaló que MySpace permitía a sus miembros crear una página web personal dentro de una estructura de marcos o frames específicos, incluyendo la carga de video, y que cada vez que era observado un video publicado por un miembro, se difundían los anuncios publicitarios sobre los cuales MySpace obtenía beneficios económicos. Estos elementos del servicio de MySpace convencieron al jurado de que actuaba como un editor $y$, por lo tanto, fue declarado responsable por el contenido infractor publicado por sus miembros.

28 United States Code, Title 17, Section 512(c) (1998). Digital Millennium Copyright Act (DMCA).

29 Tribunal de Grande Instance de Paris (2007). Asunto: Lafesse vs. MySpace. 
En el mismo sentido, otro tribunal francés encontró responsable a Dailymotion por alojar material infractor de uno de sus usuarios. Dailymotion es un sitio web para libre intercambio de videos, similar a YouTube, que permite a los usuarios guardar y cargar archivos. Los productores de la película Joyeux Noël demandaron a Dailymotion por infracción de derechos de autor, debido a que la película se encontraba disponible en ese sitio web sin el correspondiente permiso. Partiendo de la decisión reciente contra MySpace ${ }^{30}$, los productores de la película argumentaron que Dailymotion debía ser tratado del mismo modo, es decir, como un editor. Dailymotion sostuvo que se trataba de un proveedor de hosting y no tenía ninguna obligación de monitorear o buscar hechos o circunstancias de infracción en su sitio web.

El tribunal de primera instancia de París dictaminó que debido a que Dailymotion no había causado que la película se publicara en su página web, no se le podía considerar como un editor. Sin embargo, el tribunal encontró a Dailymotion responsable de la infracción de derechos de autor sobre la base de que era consciente de la presencia de contenidos ilegales en su sitio web. El tribunal consideró que el éxito de Dailymotion dependía de la disponibilidad de obras conocidas y protegidas por el derecho de autor, situación que atraía a un público más amplio y, por consiguiente, generaba mayores ingresos por publicidad. El tribunal además consideró que Dailymotion proporcionaba a sus usuarios los medios para cometer infracciones, y el puerto seguro dado a los intermediarios, según el tribunal, no se aplicaba en circunstancias en que el proveedor de alojamiento inducía a actos de infracción. El tribunal afirmó que Dailymotion era consciente del contenido infractor de su sitio web y, por tanto, tenía la obligación jurídica de controlar dicha actividad ilegal.

\section{Caching}

El caching en internet se define como el almacenamiento inmediato y temporal de los datos que se transmiten a través de los intermediarios ${ }^{31}$. El caching ayuda a reducir la congestión del intermediario de internet y a

30 Tribunal de Grande Instance de Paris (2007). Asunto: Nord-Ouest Prod. vs. S.A. Dailymotion. 2007.

31 Veáse: http://en.wikipedia.org/wiki/Cache 
aumentar la velocidad de descarga de posteriores solicitudes que recaen sobre los mismos datos. El intermediario puede, a través de un proceso automatizado, conservar copias, por un tiempo limitado, del material que transmiten sus suscriptores a otros usuarios del sistema, a fin de que las solicitudes posteriores del mismo material puedan ser enviadas mediante la transmisión de la copia conservada, en lugar de recuperar el material de la fuente original.

A nivel legislativo, el artículo 13 de la Directiva 2000/31 de la Unión Europea establece todos los requisitos de inmunidad de los intermediarios de internet para el almacenamiento en caché, lo que le implica las siguientes condiciones: a) no modifique la información; b) cumpla las condiciones de acceso a la información; c) cumpla las normas relativas a la actualización de la información, establecidas de manera ampliamente reconocida y utilizada por el sector; d) no interfiera en la utilización lícita de tecnología ampliamente reconocida y utilizada por el sector, con el fin de obtener datos sobre la utilización de la información; y e) actúe con prontitud para retirar la información que haya almacenado, en cuanto tenga conocimiento efectivo del hecho de que esta ha sido retirada del lugar de la red en que se encontraba inicialmente, de que se ha imposibilitado el acceso a dicha información o de que un tribunal o una autoridad administrativa ha ordenado retirarla o impedir que se acceda a ella.

En la legislación de Estados Unidos también se encuentran menciones claras y explícitas al respecto. En esta norma, los intermediarios de internet tampoco son responsables por vulneración de derecho de autor por parte de terceros, siempre y cuando sus actividades no alteren en medida alguna el contenido que se transmite (DeBeer \& Clemmer, 2008, pp. 380-381). Entre las condiciones generales que se pueden encontrar se nombran las siguientes: a) los intermediarios de internet no deben modificar los datos almacenados en el caché; b) deben cumplir con las reglas estándares de la industria con respecto a la restauración, recarga y cualquier otra actualización del caché almacenado; c) no deben interferir con la tecnología que recoge y devuelve información sobre los datos de uso; d) deben limitar el acceso a los destinatarios de los datos de caché; y e) los intermediarios de internet deben eliminar inmediatamente los 
contenidos infractores de derechos de autor que hayan sido puestos bajo su conocimiento.

Aunque a la fecha presente no exiten muchos casos judiciales que traten el catching, es importante mencionar una demanda presentada en Canadá32 por parte de la Sociedad de Compositores, Autores y Editores (SOCAN) contra la Asociación Canadiense de Proveedores de Internet (CAIP) ${ }^{33}$. SOCAN, la demandante, presentó acción judicial exigiendo percibir regalías por parte de los proveedores de servicios de internet situados en Canadá, porque estos violaban el derecho exclusivo conferido por la ley al titular del derecho de autor, al autorizar y comunicar la obra al público mediante su servicio de comunicaciones en internet.

En el 2004, el Tribunal Supremo de Canadá determinó, entre otros argumentos esgrimidos, que el intermediario de internet que no se dedicara a una actividad relacionada con el contenido de la comunicación, sino que se limitara a ser "un agente" que permitiera a otros comunicarse, no tenía responsabilidad frente a los titulares de los derechos de autor, conforme a la sección 2.4 (literales 1 y b) de su Ley de Copyright. El tribunal afirmó que lo que caracteriza, entre otras cosas, a tal "agente" es la ignorancia del contenido ilícito y la imposibilidad de vigilar la enorme cantidad de archivos que circulan en internet.

Para el tribunal, el caching no tiene ninguna incidencia sobre el contenido; también, conforme al artículo 2.4 (literales 1 y b) de la Ley, no debería tener ningún efecto jurídico sobre la comunicación efectuada entre el proveedor de contenido y el usuario final. El hecho de que un proveedor de servicios de internet sepa que alguien podría violar el derecho de autor gracias a una tecnología no equivale necesariamente a la autorización de esta violación, pues hace falta demostrar que el interesado aprobó, sancionó, permitió, favoreció o incentivó el comportamiento ilícito. Por lo anterior, el tribunal desestimó la demanda de la SOCAN.

32 En Canadá no se establece ninguna norma específica en sus estatutos. Sin embargo, fue objeto de estudio judicial.

33 Sentencia SOCAN vs. CAIP (2004). 2 S.C.R. 427, 2004 SCC 45. Pueden verse extractos de la sentencia publicada por la Unesco en http://portal.unesco.org/culture/es/files/23815/11515055391socan_sp.pdf/ socan_sp.pdf 


\section{Nuevos modelos legislativos}

Si bien se ha visto que los intermediarios en las legislaciones mundiales actúan de una manera pasiva y neutral antes de tener conocimiento de contenidos ilegítimos en sus sistemas, actualmente algunas decisiones judiciales, iniciativas político-legislativas y negociaciones multilaterales ${ }^{34}$ están haciendo que en algunos países se exija una labor más activa a los intermediarios de internet, en relación con la protección y observancia del derecho de autor. Sistemas como el de Francia y el Reino Unido han implementado estos nuevos acercamientos. Es importante mencionar que estos nuevos modelos legislativos han generado fuertes oposiciones de los usuarios y la sociedad civil, en tanto se afirma que se comprometen los derechos humanos al coartar la libertad de expresión y vulnerar las leyes de privacidad, protección de datos y el debido proceso.

\section{Francia}

La Ley de Promoción de la Distribución de Obras Creativas y la Protección de los Derechos en Internet (o Ley HADOPI ${ }^{35}$ ) fue introducida a la Asamblea Nacional francesa durante el año 2009, en tanto medio para controlar y regular el acceso a internet y fomentar el cumplimiento de las leyes de derecho de autor. El proyecto de ley fue rechazado inicialmente, pero el gobierno francés solicitó la reconsideración del proyecto de ley y finalmente fue aprobado por la Asamblea Nacional francesa el 12 de mayo 2009, y el 13 de mayo de 2009 por el Senado francés. Sin embargo, el 10 de junio el Consejo Constitucional declaró la parte principal del proyecto de ley como inconstitucional, al considerar que la ley violaba la Declaración

34 Anti-Counterfeiting Trade Agreement (ACTA) o Acuerdo Comercial Anti-Falsificación: este acuerdo es una propuesta comercial plurilateral, según sus promotores, en respuesta al incremento de los bienes falsificados y obras protegidas por copyright pirateadas en el mercado global. El ámbito de ACTA es amplio, incluyendo la falsificación de bienes físicos, así como la distribución en internet y en las nuevas tecnologías de la información. ACTA es un nuevo marco legal internacional al cual los países pueden adherirse voluntariamente y de manera independiente a las instituciones internacionales existentes, como la Organización Mundial del Comercio (OMC), la Organización Mundial de la Propiedad Intelectual (OMPI) o la Organización de las Naciones Unidas (ONU). A la fecha de publicación del presente artículo, el acuerdo fue firmado, el 1 de octubre de 2011, por Australia, Canadá, Corea del Sur, Japón, Estados Unidos de América, Marruecos, Nueva Zelanda y Singapur, en Tokio (Japón). Otros países que habían participado en la negociación, como Mexico, Suiza y la Unión Europea (UE), no firmaron el acuerdo.

35 El texto de la ley se puede encontrar en http://www.hadopi.fr/actualites/textes-juridiques/ code-de-la-propriete-intellectuelle 
de los Derechos del Hombre y del Ciudadano de 1789, en especial la presunción de inocencia, la separación de poderes y la libertad de expresión. En respuesta se presentó un proyecto de ley modificado, y el 22 de octubre de 2009, el Consejo Constitucional aprobó el texto, bajo la condición de que los casos en que se fuera a cancelar una cuenta de acceso de internet de un suscriptor infractor se debían someter a revisión judicial.

En términos generales, la ley establece que en el momento en que se recibe una reclamación del titular de derecho de autor por el uso de contenido infractor en internet, la agencia gubernamental francesa HADOPI puede iniciar un procedimiento denominado "los tres strikes", comprendido por tres etapas. En la primera etapa se envía un mensaje de correo electrónico de advertencia al presunto infractor y suscriptor de acceso a internet a partir de su dirección IP. En este caso, el proveedor de servicio de internet ISP al que se encuentra suscrito el presunto infractor está en la obligación de vigilar la conexión de internet de su suscriptor. Si en los seis meses siguientes desde la primera reclamación el titular de los derechos sospecha la reincidencia del suscriptor, el titular, el proveedor de servicios de internet o HADOPI invocan la segunda etapa del procedimiento de reclamación.

En la segunda etapa se envía una carta certificada al suscriptor presuntamente infractor, con un contenido similar al mensaje de correo electrónico inicial, a fin de que no continúe con la actividad infractora. Si durante el año siguiente el presunto infractor no da cumplimiento a lo solicitado en la comunicación, el titular de los derechos, el proveedor de internet o HADOPI pueden invocar el tercer paso del procedimiento de reclamación: la obligación de suspender el acceso a Internet del suscriptor infractor por un periodo de entre dos meses y un año.

Este suscriptor entra a hacer parte de una lista de suspensiones, y a los otros proveedores de servicio de internet se les impone la prohibición de proporcionar una conexión a internet al suscriptor sancionado. Sin embargo, la suspensión del servicio no exime al suscriptor del pago de las mensualidades, y este es obligado a cumplir con todos los gastos y costes derivados de la cancelación del servicio. Apelar ante un tribunal de justicia solo es posible durante esta tercera etapa, y la carga de la prueba 
se encuentra en cabeza del suscriptor suspendido. Es importante tener en cuenta que esta reclamación no excluye la posibilidad de que el titular de los derechos pueda iniciar otras acciones legales bajo la ley francesa de propiedad intelectual.

\section{Reino Unido}

En el 2009, el gobierno inglés introdujo la Ley de Economía Digital ${ }^{36}$, que tuvo primera lectura en la Cámara de los Lores el 19 de noviembre de 2009, segunda lectura el 2 de diciembre de 2009 y tercera lectura el 15 de marzo de 2010. La ley implementa muchos aspectos del "Digital Britain Report" ${ }^{\prime 37}$, cubriendo una amplia gama de áreas, tales como la violación de los derechos de autor en línea y la seguridad digital. Dentro de los temas incluidos en la ley, las secciones 3 a 16 son las que resultan de mayor impacto para la observancia del derecho de autor en internet, por cuanto se imponen diversas obligaciones a los ISP, encaminadas a la reducción de la infracción de los derechos de autor en linea ${ }^{38}$.

Al respecto, las disposiciones de la ley establecen un sistema que apunta, primero, a aumentar la facilidad de localizar y demandar a los infractores persistentes; $y$, segundo, a establecer un sistema para introducir "medidas técnicas", a fin de reducir el ancho de banda o terminar las conexiones de los infractores. También crea un nuevo proceso judicial para tramitar las apelaciones. El nuevo proceso, que entrará en vigor una vez el código regulador de Ofcom ${ }^{39}$ sea aprobado por el Parlamento, contempla diversas etapas en la reclamación, siendo la primera la creación de una lista elaborada por los titulares de derecho de autor, en la cual se recogerían las direcciones de IP de suscriptores que ellos creen que han vulnerados sus derechos ${ }^{40}$.

36 Veáse el texto en http://www.publications.parliament.uk/pa/cm200910/cmbills/089/10089.i-iii.html

37 Véase http://news.bbc.co.uk/2/shared/bsp/hi/pdfs/16_06_09digitalbritain.pdf

38 Al respecto, ver la Ley de Comunicaciones, secciones 124A a 124M.

39 Regulador y autoridad de competencia para las industrias de comunicaciones del Reino Unido. Ver en http://www.ofcom.org.uk/

40 Este tipo de información se puede recoger a partir de las direcciones de IP que se conectan para descargar contenidos por medio de peer to peer. 
En la siguiente etapa del proceso se envía el número IP al proveedor de servicios de internet en que se encuentre suscrita esa dirección, junto con un informe explicando la infracción de derecho de autor. En respuesta, el proveedor de servicios de internet deberá enviar una notificación al suscriptor, comunicando la reclamación que han presentado los titulares de los derechos de autor ${ }^{41}$ En la última etapa del procedimiento, y ante la reincidencia en la infracción, el titular de derechos solicita al ISP una lista de los infractores suscritos a su servicio. Esta lista contiene información anónima de todos los infractores que han llegado al umbral máximo de vulneraciones establecido en el Código Ofcom. En este punto del proceso, el titular de los derechos puede solicitar a un juez una orden judicial para identificar algunos o todos los suscriptores de la lista y, una vez entregada, iniciar los procesos legales y judiciales correspondientes por la violación de derecho de autor.

Esta legislación, al igual que la de Francia, ha planteado inquietudes respecto a la limitación del derecho a la protección de los datos personales, así como del derecho al respeto del secreto de las comunicaciones. La Comisión Europea ha declarado, en algunas ocasiones, que la posibilidad de conservar el anonimato resulta esencial para preservar los derechos fundamentales a la vida privada en el ciberespacio ${ }^{42}$. Por su parte, la implantación de un sistema de filtrado y de bloqueo de las comunicaciones electrónicas -como el que se exige-tampoco puede dejar de tener consecuencias para el derecho al respeto de la correspondencia y de las disposiciones de la Directiva 2002/58, relativas al secreto de las comunicaciones electrónicas, interpretado a la luz del artículo 8 del Convenio Europeo para la Protección de los Derechos Humanos y de las Libertades Fundamentales, como también en relación con la jurisprudencia pertinente del Tribunal Europeo de Derechos Humanos.

41 Las condiciones bajo las cuales es necesario enviar la comunicación no están especificadas en la Ley, pero se deberá determinar en el Código Regulador de Ofcom.

42 Comunicación de la Comisión al Consejo, al Parlamento Europeo, al Comité Económico y Social y al Comité de las Regiones: creación de una sociedad de la información más segura, mediante la mejora de la seguridad de las infraestructuras de información y la lucha contra los delitos informáticos. (E-Europe [2002]. Com. 890 final, especialmente p. 23). 
Estas nuevas leyes del Reino Unido y de Francia también han sido adoptadas por Nueva Zelanda, España, Malasia y Corea del Sur; en términos generales se puede apreciar que todas estas normas buscan obtener la suspensión de las cuentas de internet de los suscriptores infractores recurrentes. Es una tendencia en incremento a nivel mundial, y posiblemente los miembros del acuerdo $\mathrm{ACTA}^{43}$ que aún no han consagrado legalmente esta clase de disposiciones, como lo son Australia, Canadá, Japón, Estados Unidos, Marruecos y Singapur, modificarán sus legislaciones, a fin de reducir el alcance de los puertos seguros de los intermediarios y obligarlos a implementar una observancia más activa del derecho de autor en internet.

\section{Conclusiones}

El cambio de las normas internacionales para la observancia del derecho de autor ha sido representativo en los últimos años y ha sido impulsado principalmente por dos factores: el primero se debe al avance de los nuevos medios tecnológicos para la creación y el uso de obras protegidas en internet; el segundo factor es la creciente importancia económica de los bienes y servicios ofrecidos en internet en el ámbito del comercio internacional, en el cual, sin duda alguna, tienen un papel muy representativo los derechos de propiedad intelectual.

Hay un tema recurrente en las legislaciones de Europa y Estados Unidos que obliga a los intermediarios de internet que proporcionan la transmisión o el alojamiento web para actuar pasivamente y de forma neutral, hasta tanto no les sea comunicada una presunta vulneración por derecho de autor o conexos por parte del titular de los derechos. Se encuentra que ninguna de las normas estudiadas obliga a los intermediarios a supervisar el contenido que se transmite o se aloja, como tampoco existe algún estatuto en que se les exija realizar grandes esfuerzos para evitar el contenido infractor de derecho de autor que se aloje o transite a través de sus redes.

43 Ver supra, cita 45 
En los ultimos años se evidencia una nueva tendencia legislativa y judicial en que se exige una labor más activa por los intermediarios de internet para la adecuada observancia de los derechos de autor en línea. Actualmente, algunas decisiones judiciales, iniciativas político-legislativas y negociaciones multilaterales están haciendo que en algunos países se involucre más a los intermediarios de internet en el respeto de estos derechos. Los más recientes sistemas legales, como el de Francia, Reino Unido, Nueva Zelanda, España, Malasia y Corea del Sur, así como las mencionadas decisiones de tribunales en Alemania, Bélgica y Francia, están moldeando a los nuevos protectores del derecho de autor en el mundo.

Sin embargo, las nuevas legislaciones de Francia o Reino Unido para la protección del derecho de autor en internet han planteado inquietudes frente a la limitación del derecho a la protección de los datos personales, así como del derecho al respeto del secreto de las comunicaciones. Como se puede apreciar en el presente escrito, la Comisión Europea ha declarado, en algunas ocasiones, que la posibilidad de conservar el anonimato resulta esencial para preservar los derechos fundamentales a la vida privada en el ciberespacio; por su parte, la implantación de un sistema de filtrado y de bloqueo de las comunicaciones electrónicas puede vulnerar el derecho al respeto de la correspondencia y derecho al secreto de las comunicaciones digitales.

En el mismo sentido, las nuevas normas para la protección y observancia del derecho de autor en internet también muestran fuertes implicaciones para la sociedad civil y, particularmente, para los usuarios de internet. Decisiones como la del Consejo Constitucional francés o la consulta prejudicial enviada al tribunal judicial de Luxemburgo evidencian situaciones legales y judiciales en que se debe establecer en qué medida la legislación autoral puede comprometer los derechos humanos, o cómo se resuelve el conflicto entre el derecho de autor cuando se ve enfrentado a la libertad de expresión, al debido proceso y a las leyes de privacidad. 


\section{Referencias}

A\&M Records vs. Napster, 239 F.3d 1004.

Blakeney, M. (2001). Enforcement and border control of intellectual property rights under the TRIPS Agreement. In Border control of intellectual property rights. London: Street \& Maxwell.

Cámara de Comercio Internacional (1998). Enforcement measures against counterfeiting and piracy: an internacional survey. París: ICCC Publishing.

Cikato, M. (1987). Negociar y golpear: nueva estrategia para la propiedad intelectual. En Derechos intelectuales. Buenos Aires: Astrea.

Conclusiones del Abogado Pedro Cruz Villalón presentadas al tribunal de justicia de Luxemburgo (2011, abril). Recuperado de http://eur-lex. europa.eu/LexUriServ/LexUriServ.do?uri=CELEX:62010CC0070:ES: HTML\#Footref7

Correa, C. M. (2001). Intellectual property rights, the WTO and the developing countries. Nueva York: Zed Books.

Corte del Distrito de Bruselas (2007, 29 de junio). Sentencia No. 04/8975/A.

DeBeer, J. \& Clemmer, C. (2008). Global trends in online copyright enforcement: a non neutral role for network intermediaries? Canadá.

Digital Millenium Copyright Act (DMCA). Estados Unidos.

Franchise Council of Australia (2005). Kazaa, 1242, Australia.

Izquierdo, A. y Palacio, G. (2011, julio-diciembre). Un acercamiento comparado sobre la implementación de los ADPIC para la observancia de los derechos de propiedad intelectual en Europa, Estados Unidos y Colombia. Revista de Derecho Privado, 46. Bogota: Universidad de los Andes. 
Observancia de Propiedad Intelectual (Intellectual Property Watch) (s.f.). Recuperado de http://www.ip-watch.org/weblog/index.php?p=602

Organisation for Economic Cooperation and Development (OECD) (2010, abril). The Economic and social role of internet intermediaries. Recuperado de http://www.oecd.org/internet/interneteconomy/44949023.pdf

Organización Mundial del Comercio (OMC) (1994, 15 de abril). Acuerdo de la OMC sobre los Aspectos de los Derechos de Propiedad Intelectual relacionados con el Comercio (ADPIC).

Organización Mundial de la Propiedad Intelectual (1883, 20 de marzo). Convenio de París (aprobado en Colombia mediante la Ley 178 de 1994).

Organización Mundial de la Propiedad Intelectual (1886, 9 de septiembre). Convenio de Berna para la Protección de Obras Literarias y Artísticas (aprobado en Colombia mediante la Ley 33 de 1987).

Organización Mundial de la Propiedad Intelectual (1961, 26 de octubre). Convenio de Roma sobre la Protección de los Artistas Intérpretes o Ejecutantes, los Productores de Fonogramas y los Organismos de Radiodifusión (aprobado en Colombia mediante la Ley 48 de 1975).

Organización Mundial de la Propiedad Intelectual (1996, 20 de diciembre). Tratado OMPI en Derecho de Autor (WCT).

Organización Mundial de la Propiedad Intelectual (1996, 30 de diciembre). Tratado de la OMPI sobre Interpretación o Ejecución y Fonogramas (WPPT).

Organización Mundial de la Propiedad Intelectual (2004). WIPO intellectual property handbook ( $2^{\mathrm{a}}$. ed.). Ginebra.

Parlamento Europeo y del Consejo (2000, 8 de juniio). Directiva 2000/31/ CE. Diario Oficial, No. L178. 
Pirate Bay (Svea hovrätt) (2010, 26 de noviembre). No. B 4041-09.

Propuesta del Parlamento Europeo y del Consejo Directivo en medidas penales para la observancia de los Derechos de Propiedad Intelectual (2005, 12 de agosto). Recuperado de http://europa.eu.int/eur-lex/lex/ LexUriServ/site/en/com/2005/com2005_0276en01.pdf

Ricolfi, M. (2004). The proposed IP enforcement directive: tough on legitimate competitors, weak against pirates. Revista Italian Intellectual Property, año II, Fasc. 1, Milano Giufrè Editore.

Sike, A. (2005). Effective enforcement mechanisms of intellectual property rights vis-a-vis economic development: Zambian case study. En Collection of research papers WIPO. Ginebra: Organización Mundial de la Propiedad Intelectual (OMPI).

Steward, T. (1993). The GATT Uruguay Round, a negotiating history (19861992) (vol. III). Boston: Kluwer.

Supreme Court of the United States (2005). Metro-Goldwyn-Mayer Studios vs. Grokster, 125 S. Ct. 2764.

Tribunal de Grande Instance de Paris (2007). Asunto: Lafesse vs. MySpace.

Tribunal de Grande Instance de Paris (2007). Asunto: Nord-Ouest Prod. vs. S.A. Dailymotion.

Tribunal Supremo de Canadá (2004). Sentencia SOCAN vs. CAIP, S.C.R. 427, S.C.C. 45. Recuperado de http://portal.unesco.org/culture/es/ files/23815/11515055391socan_sp.pdf/socan_sp.pdf

U.S. Court of Appels for the Ninth Circuit (2001). Wu, Tim (2004). Copyright's Communications Policy. Michigan Law Review, 103. 